\title{
A176 MOLECULAR MECHANISMS OF PERIPHERAL CD25 REGULATORY T CELL (TREG) DEVELOPMENT
}

I Prots, A Skapenko, P E Lipsky, H Schulze-Koops Division of Rheumatology, Med. Poliklinik, University of Munich, Munich, Germany and Autoimmunity Branch, NIAMS, Bethesda, MD, USA

\subsection{6/ard.2010.129668m}

Compromised development and/or function of CD25 regulatory T cells (Tregs) is of central importance for the development of autoimmune diseases. The mechanisms that regulate the generation of peripheral Tregs are, however, largely unknown. To gain insights into Treg differentiation on a molecular level, the authors investigated gene expression profiles of Tregs at different developmental stages.

Human Tregs were generated in vitro from CD4 CD25-naive $T$ cells in the presence of autologous feeder cells and interleukin (IL) 4 as described previously by our group. Total RNA was isolated at the start and from purified CD25 and CD25 T cells at days 3, 5, 7 and 10 of culture and used for DNA microarrays. CD25 T cells generated in the absence of IL4 constituted activated effector $\mathrm{T}$ cells (Teffs) lacking regulatory capacity and were therefore used to control for Treg-specific gene expression. Differentially expressed transcripts in Tregs and Teffs versus CD25 T cells were determined at each time point by the microarray data analysis using GeneSpring GX10 software.

A decrease in the number of regulated transcripts was observed in Tregs during the development compared with the control cell population. Analysis of the biological processes and pathways differentially regulated between Tregs and the control population revealed an activated and proliferative state of Tregs at early developmental stages, progressing to a transcriptionally more quiescent phenotype at later time points. A lack of significant overlaps of $\mathrm{T}$ cell pathways present in Tregs at late development and in Teffs suggests dichotomy of the two populations, in particular of mature cells. The transcription factor MAFF, the chemokine receptor CCR2, the enzyme prostaglandin D synthase and the neuromediator npT1 were identified as Treg-specific genes at different developmental stages. Expression of npT1 transcript was most pronounced in mature Tregs. Moreover, transcription factors of the Fox $O$ family and FoxM1 were identified as molecular pathways specifying Treg development and activation of Teffs. These data offer new insights into Treg development in the periphery and might provide new targets for therapies of disorders caused by dysregulation of Tregs. 